\title{
Extracto del documento de consenso sobre el diagnóstico y tratamiento de la faringoamigdalitis aguda**
}

\author{
Excerpt from the consensus document on the diagnosis \\ and treatment of acute pharyngotonsillitis
}

Roi Piñeiro Pérez, ${ }^{*, \ddagger}$ Francisco Hijano Bandera, ${ }^{\S}$ Fernando Álvez González,* Ana Fernández Landaluce," Juan Carlos Silva Rico," Carlos Pérez Cánovas," Cristina Calvo Rey,* María José Cilleruelo Ortega,* Grupo de trabajo de infecciones de manejo ambulatorio de la Sociedad Española de Infectología Pediátrica

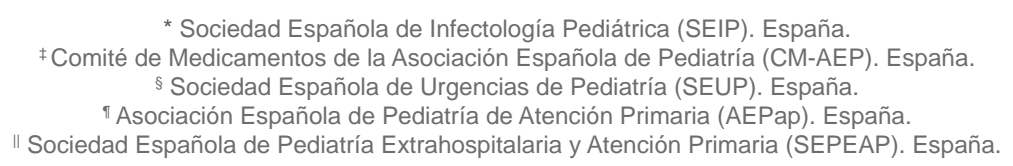

RESUMEN

La faringoamigdalitis aguda (FAA) es una de las enfermedades más comunes en la infancia. La etiología más frecuente es vírica. Entre las causas bacterianas, el principal agente responsable es Streptococcus pyogenes o estreptococo beta hemolítico del grupo A (EbhGA). Las escalas de valoración clínica son una buena ayuda para seleccionar a qué niños se deben practicar las técnicas de detección rápida de antígeno estreptocócico (TDR) y/o el cultivo de muestras faringoamigdalares. Sin su empleo, se tiende al sobrediagnóstico de FAA estreptocócica con la consiguiente prescripción innecesaria de antibióticos, muchas veces de amplio espectro. Los objetivos del tratamiento son: acelerar la resolución de los síntomas, reducir el tiempo de contagio y prevenir las complicaciones supurativas locales y no supurativas. Lo ideal es tratar sólo los casos confirmados. En caso de no disponibilidad de la TDR, o en algunos casos ante un resultado negativo, se recomienda obtener cultivo e iniciar tratamiento a la espera de los resultados, sólo si la sospecha clínica es alta. Los antibióticos de elección para el tratamiento de la FAA estreptocócica son penicilina y amoxicilina. Amoxicilina-clavulánico no está indicado de forma empírica en la infección aguda. Los macrólidos tampoco son un tratamiento de primera elección; su uso debe reservarse para pacientes con alergia inmediata a penicilina o como tratamiento

\begin{abstract}
Acute pharyngotonsillitis is one of the most common childhood illnesses. The most common aetiology is viral. Among the bacterial causes, the main agent responsible is Streptococcus pyogenes or beta haemolytic group A streptococcus. Clinical rating scales are a good aid in selecting children for rapid streptococcal antigen detection (RSAD) and/or culture of pharyngotonsillary samples. Without their use, there is a tendency to overdiagnose streptococcal acute pharyngotonsillitis, leading to unnecessary prescription of antibiotics, often broad-spectrum. The aims of treatment are: to accelerate the resolution of symptoms, to reduce the time of infection and to prevent local suppurative and non-suppurative complications. Ideally, only confirmed cases should be treated. In case of unavailability of RSAD, or in some cases, in case of a negative result, it is recommended to obtain culture and initiate treatment pending results, only if clinical suspicion is high. The antibiotics of choice for the treatment of streptococcal AFA are penicillin and amoxicillin. Amoxicillin-clavulanic acid is not indicated empirically in acute infection. Macrolides are also not a first-choice treatment; their use should be reserved for patients with immediate penicillin allergy or as eradicant treatment, when indicated. It is urgent and a priority in our
\end{abstract} WwW.medigraphic.org. mx

Citar como: Piñeiro PR, Hijano BF, Álvez GF, Fernández LA, Silva RJC, Pérez CC et al. Extracto del documento de consenso sobre el diagnóstico y tratamiento de la faringoamigdalitis aguda. Rev Latin Infect Pediatr. 2021; 34 (2): 62-72. https://dx.doi.org/10.35366/100544

Recibido: 03-06-2021. Aceptado: 06-06-2021.

** Piñeiro Pérez R, Hijano Bandera F, Álvez González F, Fernández Landaluce A, Silva Rico JC, Pérez Cánovas C et al. Documento de consenso sobre el diagnóstico y tratamiento de la faringoamigdalitis aguda. An Pediatr (Barc). 2011; 75: 342.e1-342.e13. doi: 10.1016/j.anpedi.2011.07.015

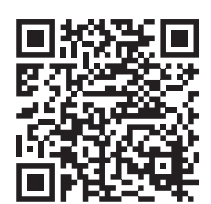


Rev Latin Infect Pediatr. 2021; 34 (2): 62-72

erradicador en los casos indicados. Es urgente y prioritario en nuestro país adecuar la prescripción de antibióticos a la evidencia científica disponible.

Palabras clave: Antibióticos, adecuación, niños, documento de consenso, diagnóstico, uso racional, Streptococcus pyogenes, amigdalofaringitis, tratamiento.

\section{DEFINICIÓN Y ETIOLOGÍA}

La faringoamigdalitis aguda (FAA) se define como un proceso agudo febril, de origen generalmente infeccioso, que cursa con inflamación de las mucosas de la faringe y/o las amígdalas faríngeas, en las que se puede objetivar la presencia de eritema, edema, exudados, úlceras o vesículas. . $^{1-4}$

La etiología más frecuente es vírica. ${ }^{1-6}$ Entre las bacterias, la principal es Streptococcus pyogenes o estreptococo beta hemolítico del grupo A (EbhGA), responsable de $30-40 \%$ de las FAA que se observan en niños de tres a 13 años, de $5-10 \%$ en niños entre los dos y tres años y sólo 3-7\% en menores de dos años..$^{1-6}$ Aunque ocurran, las FAA por EbhGA son muy raras en niños menores de dos años, e insólitas en los menores de 18 meses. La mayoría son probablemente portadores de EbhGA que padecen una infección vírica del tracto respiratorio superior y no requieren tratamiento antibiótico. ${ }^{1-3,7} \mathrm{Se}$ transmite por vía respiratoria a través de las gotas de saliva expelidas al toser, estornudar o cuando hablan personas infectadas. También se han descrito brotes transmitidos por contaminación de los alimentos o el agua; por el contrario, los fómites no desempeñan un papel importante como fuente de contagio. ${ }^{1-3}$

La edad del niño, la estación del año y el área geográfica en la que vive influyen en el tipo de agente implicado. En la Tabla 1 se muestran los microorganismos que pueden producir una FAA. En $30 \%$ de los casos no se identifica ningún patógeno. ${ }^{1-3,7}$

\section{CLÍNICA}

En la mayoría de los casos es difícil diferenciar, basándose en la clínica, entre etiología vírica y estreptocócica de FAA; no obstante, son orientadoras algunas características ${ }^{2,7}$ que se reflejan en la Tabla 2.

Son sugestivos de origen estreptocócico el dolor de garganta de comienzo brusco, la fiebre, el malestar general y la cefalea; también el dolor abdominal, las náuseas y los vómitos, especialmente en los niños más pequeños. La existencia de petequias country to adapt antibiotic prescribing to the available scientific evidence.

Keywords: Antibiotics, appropriateness, children, consensus document, diagnosis, rational use, Streptococcus pyogenes, tonsillopharyngitis, treatment.

en el paladar no es exclusiva de la FAA por EbhGA, pues también se han descrito en la rubéola y en las infecciones por herpes simple y virus de Epstein-Barr. Más específica es la presencia en el paladar blando y/o paladar duro de pequeñas pápulas eritematosas con centro pálido denominadas lesiones donuts y que sólo se han señalado en la FAA por EbhGA. ${ }^{1-3,7}$ Por lo general, la infección es autolimitada aun sin tratamiento antibiótico; la fiebre remite en tres a cinco días y el dolor de garganta en una semana, pero persiste el riesgo potencial de que se presenten complicaciones. ${ }^{2}$

En menores de tres años, la FAA por EbhGA se manifiesta de un modo distinto que en niños mayores. En lugar de un episodio agudo de faringitis suelen presentar un comienzo más indolente, con inflamación faringoamigdalar, congestión y secreción nasal mucopurulenta persistente, fiebre (no elevada), adenopatía cervical anterior dolorosa y a veces lesiones de tipo impétigo en narinas. A este complejo de síntomas, que suele ser prolongado en el tiempo, se le conoce con el nombre de estreptococosis, fiebre estreptocócica o nasofaringitis estreptocócica, clínicamente difícil de distinguir de las infecciones virales tan frecuentes en este grupo de edad.

A cualquier edad, la presencia de conjuntivitis, rinorrea, afonía, tos y/o diarrea es un hallazgo sugestivo de un origen vírico de la infección. . $^{1-3,7,8}$ También se pueden evidenciar, además de eritema e inflamación faringoamigdalar, aftas, vesículas o ulceraciones, o exudado de cuantía variable según el tipo de virus responsable. En las FAA por adenovirus, más frecuentes en menores de tres años, el cuadro clínico típico se presenta con fiebre muy elevada, mayor de $39{ }^{\circ} \mathrm{C}$, asociada con exudado amigdalar en $50 \%$ de los casos y adenopatía cervical anterior, rinorrea, tos y/o conjuntivitis en $15-25 \%$. Debido a las características clínicas de este tipo de infección viral, capaz de mimetizar una FAA estreptocócica, es muy frecuente que el manejo terapéutico no sea adecuado. El test de detección rápida de adenovirus en exudado nasofaríngeo, en caso de estar disponible, puede ayudar a diferenciar este tipo de FAA y a evitar el uso innecesario de antibióticos. ${ }^{8}$ 
Rev Latin Infect Pediatr. 2021; 34 (2): 62-72

Tabla 1: Causas infecciosas de faringoamigdalitis aguda.

\begin{tabular}{|c|c|c|}
\hline Microorganismos & $\begin{array}{l}\text { Síndrome o enfermedad } \\
\text { Hallazgos clínicos y epidemiológicos }\end{array}$ & Incidencia (en \%) \\
\hline \multicolumn{3}{|l|}{ Virus } \\
\hline Rinovirus & Resfriado común. Predominan en otoño y primavera & 20 \\
\hline Coronavirus & Resfriado común. Predominan en invierno & $\geq 5$ \\
\hline Adenovirus & Fiebre faringoconjuntival. Predomina en verano & 5 \\
\hline Virus herpes simple tipo & Gingivoestomatitis (primoinfección): vesículas y úlceras superficiales en el paladar. & 4 \\
\hline 1 y 2 & Puede cursar con exudados faríngeos & \\
\hline Virus influenza & Gripe: fiebre, mialgias, cefalea, tos & 2 \\
\hline Virus parainfluenza & Resfriado, crup & 2 \\
\hline Virus Coxsackie A & Herpangina, enfermedad mano-pie-boca & $<1$ \\
\hline Virus de Epstein-Barr & $\begin{array}{l}\text { Mononucleosis infecciosa. Los anticuerpos heterófilos generalmente no suelen ser } \\
\text { positivos hasta la segunda semana de enfermedad, ni consistentemente positivos en } \\
\text { menores de } 4 \text { años }\end{array}$ & $<1$ \\
\hline Citomegalovirus & $\begin{array}{l}\text { Síndrome mononucleósico. A diferencia del causado por VEB: faringitis menos } \\
\text { evidente y mayor elevación de transaminasas }\end{array}$ & $<1$ \\
\hline $\begin{array}{l}\text { Virus de la inmunodeficiencia } \\
\text { humana }\end{array}$ & $\begin{array}{l}\text { Infección aguda primaria: fiebre, mialgias, artralgias, malestar general, exantema } \\
\text { maculopapular no pruriginoso, que se sigue de linfadenopatía y ulceraciones mucosas } \\
\text { sin exudado }\end{array}$ & $<1$ \\
\hline \multicolumn{3}{|l|}{ Bacterias } \\
\hline $\begin{array}{l}\text { Estreptococo beta hemolítico } \\
\text { del grupo } \mathrm{A}\end{array}$ & Faringitis, escarlatina & $15-30$ \\
\hline $\begin{array}{l}\text { Estreptococo beta hemolítico } \\
\text { grupos } \mathrm{C} \text { y } \mathrm{G}\end{array}$ & Faringitis. Serogrupos no reumatógenos & 5 \\
\hline Anaerobios & $\begin{array}{l}\text { Angina de Vincent (gingivoestomatitis necrotizante): encías muy inflamadas y } \\
\text { dolorosas, con úlceras en las papilas interdentales que sangran con facilidad. Se } \\
\text { acompaña de fiebre, malestar general, halitosis y linfadenopatías }\end{array}$ & $<1$ \\
\hline Fusobacterium necrophorum & $\begin{array}{l}\text { Síndrome de Lemierre (tromboflebitis séptica de la vena yugular interna): dolor, } \\
\text { disfagia, tumefacción y rigidez de cuello }\end{array}$ & $<1$ \\
\hline $\begin{array}{l}\text { Arcanobacterium } \\
\text { haemolyticum }\end{array}$ & $\begin{array}{l}\text { Faringitis, exantema escarlatiniforme (en superficie de extensión de los brazos). Más } \\
\text { frecuente en adolescentes. Puede cursar con exudados faríngeos }\end{array}$ & $<1$ \\
\hline Neisseria gonorrhoeae & Faringitis & $<1$ \\
\hline Treponema pallidum & Sífilis secundaria & $<1$ \\
\hline Francisella tularensis & $\begin{array}{l}\text { Tularemia faríngea. Consumo de carne poco cocinada de animales silvestres o agua } \\
\text { contaminada }\end{array}$ & $<1$ \\
\hline Corynebacterium diphtheriae & Difteria. Puede cursar con exudados faríngeos & $<1$ \\
\hline Yersinia enterocolitica & Faringitis, enterocolitis. Puede cursar con exudados faríngeos & $<1$ \\
\hline Mycoplasma pneumoniae & Bronquitis, neumonía & $<1$ \\
\hline Chlamydophila pneumoniae & Bronquitis, neumonía & $<1$ \\
\hline Chlamydophila psittaci & Psitacosis & $<1$ \\
\hline
\end{tabular}

La mononucleosis infecciosa, más frecuente en la adolescencia, cursa con fiebre, faringoamigdalitis y adenopatías dolorosas de predominio laterocervical. Cuando no se asocian signos sugerentes como hepatomegalia o esplenomegalia, es frecuente confundir esta entidad con la FAA estreptocócica y es también habitual el uso innecesario de antibióticos. La toma de antibióticos puede desencadenar un exantema maculopapular característico hasta en $90 \%$ de los casos. La hipertransaminasemia y la presencia de linfocitos activados son sugerentes de mononucleosis. Se debe confirmar la infección mediante la detección de anticuerpos heterófilos o test de PaulBunnell (monotest), más fiable en mayores de cuatro 
Rev Latin Infect Pediatr. 2021; 34 (2): 62-72

años. En niños pequeños con monotest negativo es necesario realizar la serología específica del virus. ${ }^{9-11}$

Otra forma de presentación de la infección por EbhGA es la escarlatina, que por lo común se asocia con infecciones de faringe. Cursa con exantema micropapuloso, más acentuado en los pliegues (signo de Pastia), lengua aframbuesada y descamación durante el periodo de convalecencia. ${ }^{2}$

\section{COMPLICACIONES}

Las complicaciones supurativas se presentan en $1-2 \%$ de los episodios de FAA por EbhGA no tratados con antibiótico, o bien tras un tratamiento antibiótico inadecuado o no cumplimentado. Ocurren por afectación de las estructuras contiguas o de aquéllas en las que drenan; así, pueden presentarse: celulitis

Tabla 2: Hallazgos clínicos y

epidemiológicos según etiología.

Sugestivos de infección por EbhGA

Dolor de garganta de comienzo brusco

Odinofagia

Fiebre

Exantema escarlatiniforme

Cefalea

Náuseas, vómitos, dolor abdominal

Eritema o exudados faringoamigdalares

Petequias en el paladar blando

Pequeñas pápulas eritematosas y anulares, con centro pálido (lesiones donuts) en paladar blando y/o duro

Úvula roja y edematosa

Adenopatías cervicales anteriores, aumentadas de tamaño (>

$1 \mathrm{~cm}$ ) y dolorosas

Edad: 5-15 años

Presentación en invierno o comienzos de la primavera (en

climas templados)

Historia de exposición a enfermo con FAA en las dos semanas previas

Sugestivos de infección vírica

Conjuntivitis

Rinorrea

Afonía

Tos

Diarrea

Exantemas o enantemas característicos

Hepatoesplenomegalia

Adenopatías generalizadas

EbhGA = estreptococo beta hemolítico del grupo A; FAA = faringoamigdalitis aguda.
Tabla 3: Sistema de calificación de la Infectious Diseases Society of America y de la US Public Health Service para establecer recomendaciones en guías clínicas.

Grado de la recomendación

A Buena evidencia para sostener una recomendación a favor 0 en contra del uso

B Evidencia moderada para sostener una recomendación a favor 0 en contra del uso

C Poca evidencia para sostener una recomendación

Nivel de la evidencia

I Evidencia de uno o más ensayos controlados debidamente aleatorizados

II Evidencia de uno o más ensayos clínicos bien diseñados, sin aleatorización, de estudios analíticos con cohorte 0 controlados por caso (preferentemente de más de un centro), de series múltiples reiteradas o de resultados considerables de experimentos no controlados

III Evidencia de opiniones de autoridades respetadas basadas en experiencia clínica, estudios descriptivos 0 informes de comités de expertos

y absceso periamigdalino, absceso retrofaríngeo, otitis media aguda, sinusitis, mastoiditis y adenitis cervical supurativa. ${ }^{1,2}$ En el absceso periamigdalino es típico el desplazamiento de la úvula y de la amígdala afectada hacia la línea media y el trismo de intensidad variable. La infección en estos casos suele ser polimicrobiana, con la implicación de microorganismos anaerobios como Prevotella spp., Porphyromonas spp., Fusobacterium spp. y Peptostreptococcus spp. No es fácil distinguir entre celulitis (o flemón) y absceso ya establecido.

La instauración precoz de un tratamiento antibiótico intravenoso durante la fase inicial de la celulitis puede evitar la necesidad de drenaje quirúrgico, que es en cualquier caso, el tratamiento de elección en el absceso ya formado.

Entre las complicaciones no supurativas cabe citar la fiebre reumática aguda (FRA), la glomerulonefritis postestreptocócica (GMNPE), la artritis reactiva postestreptocócica, el eritema nodoso, la púrpura anafilactoide y el síndrome PANDAS (acrónimo del inglés pediatric autoimmune neuropsychiatric disorders associated with Streptococcus [síndrome neuropsiquiátrico autoinmunitario asociado con infección por estreptococo en la edad pediátrica]). ${ }^{1-4}$ La FRA es muy poco frecuente en los países desarrollados. En menores de tres años es prácticamente inexistente antes de que haya madurado completamente su sistema inmunitario, pero se 
Rev Latin Infect Pediatr. 2021; 34 (2): 62-72

mantiene como la principal causa de enfermedad cardiaca adquirida en niños de países en desarrollo a cualquier edad. ${ }^{7}$

\section{ORIENTACIÓN DIAGNÓSTICA}

La decisión primordial y también práctica ante una FAA es averiguar si es causada por el EbhGA. Sin el empleo de los estudios microbiológicos, dada la inespecificidad de los síntomas, se tiende al sobrediagnóstico de la FAA estreptocócica, con la consiguiente prescripción innecesaria de antibióticos. ${ }^{1,2}$ Estas pruebas se recomiendan en pacientes bien seleccionados que reúnan criterios de infección estreptocócica probable y que no hayan recibido tratamiento antibiótico previo, evitándolas cuando se sospeche un origen vírico. ${ }^{2,7,12-14}$ (Grado de recomendación $A$, nivel de evidencia I, tal y como se refleja según el sistema de calificación de la Infectious Diseases Society of America y de la US Public Health Service en la Tabla 3).

Con el fin de facilitar el diagnóstico, se han propuesto varios sistemas o escalas de puntuación según las manifestaciones clínicas y datos epidemiológicos. Una de las más empleadas es la de Mclsaac, basada en los criterios de Centor, pero ponderando la edad (Tabla 4). ${ }^{15,16}$

La probabilidad de un resultado positivo en las pruebas de diagnóstico microbiológico es $\leq 3 \%$ en los pacientes que no cumplen ninguno de los criterios clínicos, y de $38-63 \%$ en los que reúnen cuatro o cinco puntos $2,15,16(\mathrm{Al})$. La presencia de síntomas virales, aunque la puntuación en la escala sea mayor

\section{Tabla 4: Criterios de Mclsaac y actitud según resultado.}

\begin{tabular}{lc} 
Criterios & Puntos \\
\hline 1. Fiebre $\left(>38^{\circ} \mathrm{C}\right)$ & 1 \\
2. Hipertrofia o exudado amigdalar & 1 \\
3. Adenopatía laterocervical anterior dolorosa & 1 \\
4. Ausencia de tos & 1 \\
5. Edad (años) & \\
- $3-14$ & 1 \\
$->15$ & 0 \\
\hline
\end{tabular}

0-1 puntos: no estudio microbiológico (riesgo de infección por EbhGA: 2-6\%). 2-3 puntos: estudio microbiológico y tratar sólo si es positivo (riesgo: 10-28\%).

4-5 puntos: estudio microbiológico e iniciar tratamiento antibiótico, si se realiza cultivo, a la espera del resultado (riesgo: $38-63 \%$ ).

EbhGA = estreptococo beta hemolítico del grupo $\mathrm{A}$.
Tabla 5: Indicaciones para solicitar pruebas

microbiológicas en la FAA (TDR y/o cultivo).

TDR:

- Puntuación $\geq 2$ en la escala de Mclsaac en ausencia de sintomatología viral

Cultivo:

- Puntuación $\geq 2$ en la escala de Mclsaac, en ausencia de sintomatología viral y cuando no exista la posibilidad de realizar TDR

- TDR negativo y presencia de alguno de los siguientes:

- Antecedentes de FRA (complicación excepcional en países desarrollados) o GMNPE, tanto en niños con FAA como en contactos domiciliarios

- Mayor incidencia en la comunidad de enfermedad estreptocócica invasiva o contacto confirmado con la misma

- Alta sospecha de origen bacteriano de la FAA a pesar de TDR negativo (valorar cultivos específicos)

- Baja sensibilidad demostrada de la TDR en el centro que realiza la prueba

- Para valorar el estado del portador

Nota: Cada centro debe validar la prueba de diagnóstico rápido que utiliza, ya que la sensibilidad puede modificarse en función de diversas variables.

FAA = faringoamigdalitis aguda; FRA = fiebre reumática aguda; GMNPE = glomerulonefritis postestreptocócica; TDR = técnicas de detección rápida de antígeno estreptocócico.

de dos puntos, anula la indicación de realizar pruebas microbiológicas. Este tipo de valoración clínica puede ser muy útil también en situaciones donde no es posible realizar el examen microbiológico, con la intención de reducir el uso innecesario de antibióticos. ${ }^{2,15,16}$

Existen dos tipos de pruebas complementarias para la detección del EbhGA: técnicas de detección rápida de antígeno estreptocócico (TDR) y cultivo de muestra faringoamigdalar. Ninguna de ellas diferencia de forma definitiva los pacientes con FAA estreptocócica verdadera de aquéllos que padecen una infección viral y son portadores de EbhGA. Sin embargo, en pacientes que están bien seleccionados por los criterios clínicos y epidemiológicos sugerentes de infección estreptocócica, estas pruebas son el mejor argumento para la indicación o no de un tratamiento antibiótico. ${ }^{1-3}$

Las TDR se basan en la extracción ácida o enzimática del antígeno carbohidrato específico de la pared celular del EbhGA y en la posterior detección de éste mediante anticuerpos específicos. La principal ventaja de las TDR es su rapidez, disponiendo del resultado 
Rev Latin Infect Pediatr. 2021; 34 (2): 62-72

en 10-20 min, lo que facilita la toma de decisiones. No son útiles para la identificación de estreptococos beta hemolíticos grupos $\mathrm{C}$ y $\mathrm{G}$. Las TDR tienen una elevada especificidad, próxima a 95\%, y una sensibilidad que puede variar entre $70-95 \%$.

Basándose en la alta especificidad, si el test es positivo, se acepta que el paciente presenta una FAA por EbhGA, no siendo precisa la confirmación mediante cultivo de muestra faringoamigdalar. En cambio, ante un resultado negativo algunos expertos sugieren realizar siempre cultivo, mientras que otros, dado el menor protagonismo y la significativa disminución de la FRA, sólo lo recomiendan cuando se dan ciertos factores de riesgo ${ }^{1,2,6,7,17-22}$ (CIII).

El cultivo es la prueba de referencia para el diagnóstico definitivo. ${ }^{1,2}$ En condiciones ideales, la sensibilidad que aporta es de $90-95 \%$ y la especificidad llega a ser de 99\%. Su mayor inconveniente es que los resultados se obtienen, en el mejor de los casos, en un plazo de 24-48 horas. Otro inconveniente es que la cuantificación del número de colonias de EbhGA no es útil para diferenciar entre infección aguda y portador, pues un escaso número de colonias se puede relacionar también con una infección verdadera. ${ }^{2,23}$ Las principales ventajas del cultivo son: el aislamiento, la identificación y la determinación de la sensibilidad antimicrobiana del EbhGA y/o de otras bacterias causantes de la FAA, vigilar la evolución de las resistencias antimicrobianas y conocer las características de los clones circulantes en cada periodo y sus serotipos, lo que permitiría diferenciar, en caso de ser necesario, entre recidivas y reinfecciones. ${ }^{1-3,24}$ Las indicaciones para solicitar pruebas microbiológicas en la FAA (TDR y/o cultivo) que se recomiendan en el presente documento de consenso se muestran en la Tabla 5 (CIII).

\section{TRATAMIENTO}

El tratamiento antibiótico está justificado porque ha demostrado una resolución más rápida de los síntomas, reducción del tiempo de contagio y transmisión del EbhGA en la familia y la escuela, y prevención de las complicaciones supurativas locales y no supurativas, aunque no existe evidencia definitiva en la protección frente a la GMNPE y el síndrome PANDAS ${ }^{1,2,7,13,14,25-38}$ (Al).

Lo ideal es tratar sólo los casos confirmados. ${ }^{1,2,7,32}$

En caso de TDR negativa (en función de la sensibilidad de cada centro y/o la presencia de factores de riesgo) o no disponibilidad de la prueba, se recomienda obtener cultivo, y sólo si la sospecha clínica es alta (4-5 puntos de los criterios de Mclsaac), se puede iniciar tratamiento antibiótico a la espera de los resultados. ${ }^{1-3,13,14,39,40}$ Otros autores, en cambio, sugieren no iniciar el tratamiento hasta conocer los resultados, dado que no existe evidencia de que su empleo precoz disminuya la infección recurrente o prevenga las complicaciones no supurativas. En este documento de consenso se considera que el tratamiento no debe demorarse en caso de estar indicado; la prevención de las complicaciones no supurativas ya no es el principal objetivo del tratamiento en países desarrollados, sino la resolución de los síntomas y la reducción del tiempo de contagio y transmisión del EbhGA (CIII).

Además de la sospecha clínica elevada, otras indicaciones aceptadas para su instauración precoz son: la presencia de un cuadro de FAA en el contexto familiar, cuando se ha confirmado el origen estreptocócico en alguno de los convivientes, y/o aunque es excepcional en nuestro medio, si existen antecedentes de FRA en el niño o en algún familiar conviviente $7,32,41$ (CIII).

Los criterios para iniciar tratamiento antibiótico en la FAA que se recomiendan en el presente documento de consenso se muestran en la Tabla 6 (CIII).

Aunque la penicilina es considerada desde hace años como el antibiótico de elección, varios estudios muestran su infrautilización en niños diagnosticados de FAA estreptocócica. Esto podría estar relacionado con las escasas presentaciones farmacéuticas de penicilina $V$ existentes junto a una mayor familiaridad con la posología de la amoxicilina, o bien con el hecho de que muchos pediatras no conocen que en nuestro país está disponible fenoximetilpenicilina benzatina oral en suspensión, fácil de administrar y con mejor sabor que fenoximetilpenicilina potásica.

España ocupa el segundo puesto en cuanto a consumo de antibióticos en la Unión Europea. La prescripción de penicilinas de espectro reducido es 20-60 veces menor que en Alemania o Suecia. Este es uno de los motivos principales por los que las guías terapéuticas en nuestro país deben dar prioridad a mensajes clave como el empleo preferente de penicilina o amoxicilina frente a otros antibióticos. Las guías actuales de tratamiento siguen recomendando como antibiótico de elección la penicilina, dado que no se ha documentado ningún aislamiento de EbhGA resistente a la misma ${ }^{2,32,39,42-45}(\mathrm{Al})$. Fenoximetilpenicilina benzatina tiene mejor perfil 
Rev Latin Infect Pediatr. 2021; 34 (2): 62-72

farmacocinético, mejor sabor que su congénere y se dispensa en suspensión. ${ }^{39}$ La administración de penicilina $G$ benzatina por vía intramuscular profunda en dosis única es dolorosa y hoy día no se recomienda. La amoxicilina en pautas de una o dos dosis diarias durante 10 días ha demostrado tasas más altas de curación clínica y erradicación del EbhGA que la penicilina V, probablemente en relación con una mayor absorción gastrointestinal y mejor tolerancia de la suspensión. Por ello, actualmente se considera este antibiótico de primera elección junto con la fenoximetilpenicilina ${ }^{2,39,45-48}(\mathrm{Al})$. Se ha constatado en nuestro país la prescripción frecuente de amoxicilina-clavulánico para tratar la FAA estreptocócica. ${ }^{49-52}$ Esta combinación no es nunca de primera elección, pues se trata de un antibiótico de amplio espectro y porque EbhGA no produce beta lactamasas ${ }^{1,2,7,39,45-48,53}(\mathrm{Al})$.

Las cefalosporinas por vía oral de primera, de segunda y tercera generación proporcionan tasas de curación clínica y bacteriológica similares e incluso superiores a la penicilina. Por este motivo, hay autores que las recomiendan como antibióticos de primera elección. ${ }^{54,55}$ Sin embargo, teniendo en cuenta estudios con mejor calidad de diseño, las diferencias en la erradicación bacteriológica con la penicilina no son superiores a $10 \%$, estimación no convincente para justificar que las cefalosporinas se indiquen como primera elección. ${ }^{39,44,45,56,57}$ Las cefalosporinas por vía oral sólo están indicadas en niños con reacción retardada a penicilina, no anafiláctica, y como posible opción en casos de

Tabla 6: Criterios para iniciar tratamiento antibiótico en la FAA.

- Casos confirmados mediante TDR o cultivo

- Casos de alta sospecha de origen bacteriano de la FAA (4-5 puntos de los criterios de Mclsaac), en caso de TDR negativa o no disponibilidad de la prueba y a la espera de los resultados del cultivo

- Presencia de un cuadro de FAA en el contexto familiar cuando se ha confirmado el origen estreptocócico en alguno de los convivientes, con independencia de los resultados de las pruebas microbiológicas

- Aunque es excepcional en nuestro medio, si existen antecedentes de FRA en el niño o en algún familiar conviviente

FAA = faringoamigdalitis aguda; TDR = técnicas de detección rápida de antígeno estreptocócico.
Tabla 7: Tratamiento antibiótico de la FAA estreptocócica.

- Primera elección. Cualquiera de las siguientes dos opciones:

- Penicilina V (fenoximetilpenicilina potásica o benzatina) durante 10 días

- Menores de 12 años y de 27 kg: 250 mg cada $12 \mathrm{~h}$

- Mayores de 12 años 0 de 27 kg: 500 mg cada $12 \mathrm{~h}$

- Amoxicilina durante 10 días

- 40-50 mg/kg/día cada $12024 \mathrm{~h}$

- Máximo 500 mg cada 12 h 01 g cada 24 h

- En caso de mal cumplimiento vía oral o vómitos

- Penicilina G benzatina, dosis única IM profunda

- Menores de 12 años y de 27 kg: 600,000 U

- Mayores de 12 años 0 de 27 kg: 1,200,000 U

- Alergia a penicilina (reacción retardada)

- Cefadroxilo durante 10 días

- 30 mg/kg/día cada 12 h. Máximo 1 g cada 24 h

- Alergia a penicilina (reacción inmediata o acelerada)

- Azitromicina durante 3 días

- 20 mg/kg/día cada 24 h. Máximo 500 mg/dosis

- Si resistencia a macrólidos, de 14 y 15 átomos (eritromicina, claritromicina y azitromicina)

- Clindamicina: 20-30 mg/kg/día cada 8-12 h, 10 días (máximo 900 mg/día)

- Josamicina: 30-50 mg/kg/día, cada 12 h, 10 días (máximo $1 \mathrm{~g} /$ día)

- Diacetato de midecamicina: 40 mg/kg/día, cada 12 h, 10 días (máximo $1.5 \mathrm{~g} / \mathrm{día}$ )

$\mathrm{FAA}$ = faringoamigdalitis aguda; $\mathrm{IM}$ = intramuscular.

recaídas frecuentes de FAA por EbhGA ${ }^{1,2,58}$ (BII) se recomienda cefadroxilo ${ }^{1,2,7,39}$ (BII).

En España se ha documentado desde 1992 un aumento significativo del número de cepas de EbhGA resistentes a los macrólidos de 14 átomos de carbono (eritromicina, roxitromicina y claritromicina) y de 15 átomos (azitromicina).39,40,49,59-62 También pueden utilizarse la claritromicina y los macrólidos de 16 átomos de carbono en el anillo lactónico como la josamicina y la midecamicina ${ }^{39,63-66}$ (BII). En caso de infección por EbhGA resistente a macrólidos de 14 y 15 átomos de carbono (confirmada o sospechada), el tratamiento depende del resultado del cultivo y antibiograma. Si la elección del antibiótico es empírica, son buenas opciones terapéuticas la clindamicina, la josamicina y la midecamicina ${ }^{1,67-69}$ (BII).

Las pautas inferiores a 10 días presentan menor porcentaje de erradicación del EbhGA, y no existe suficiente evidencia científica para recomendarlas, salvo en el caso de la azitromicina, antibiótico 
Rev Latin Infect Pediatr. 2021; 34 (2): 62-72

que debido a sus características farmacocinéticas persiste en el tejido amigdalar durante más tiem$\mathrm{po}^{32,34,70,71}(\mathrm{Al})$.

Las dosis y las pautas de los antibióticos recomendados para el tratamiento de la FAA estreptocócica en el presente documento de consenso se muestran en la Tabla 7.

\section{EVOLUCIÓN}

La mayoría de los niños presenta mejoría clínica en las primeras 48 horas tras la instauración de un tratamiento antibiótico. La posibilidad de contagio está eliminada en las primeras 24 horas de tratamiento, no está indicado realizar un cultivo ni una TDR como «prueba de curación».1,2 El primero sucede raramente en niños que, tras la prescripción de un tratamiento adecuado, siguen presentando signos y síntomas de FAA con persistencia del EbhGA en la faringe. Cuando el paciente tiene una recaída por EbhGA, menos de 15 días después de terminar el tratamiento, lo más frecuente es que se deba al mismo serotipo que causó la infección inicial. ${ }^{1,72}$ En estos casos, cabe volver a tratar con un nuevo ciclo del mismo antibiótico, administrar una dosis única de penicilina benzatina o bien otro antibiótico con mayor estabilidad frente a las beta lactamasas ${ }^{1,2,23}$ (BII).

El fracaso bacteriológico del tratamiento se refiere a la persistencia de EbhGA en la faringe de niños asintomáticos, tras un tratamiento adecuado y bien cumplimentado. Esto no significa que exista un fracaso real del tratamiento. Para valorar mejor esta colonización es preferible realizar un cultivo, pues tras el tratamiento pueden persistir en la faringe fragmentos antigénicos de EbhGA que pueden dar un resultado falso positivo con las TDR. Ningún esquema terapéutico, en particular para la FAA,

Tabla 8: Indicaciones de tratamiento del fracaso terapéutico bacteriológico y del estado de portador de EbhGA.

a. Antecedente de fiebre reumática en el niño o convivientes

b. Brotes intrafamiliares recurrentes de FAA por EbhGA

c. Enfermedad invasiva por EbhGA en el niño o convivientes

d. Portadores que viven en instituciones cerradas o con enfermos crónicos

e. Cuando se contempla realizar una amigdalectomía como último recurso de tratamiento

EbhGA = estreptococo beta hemolítico del grupo $A ; F A A=$ faringoamigdalitis aguda.
Tabla 9: Tratamiento antibiótico fracaso terapéutico bacteriológico y del estado de portador de EbhGA.

Opciones terapéuticas:

- Clindamicina durante 10 días - 20-30 mg/kg/día cada 8-12 h. Máximo 900 mg/día

- Azitromicina durante 3 días - 20 mg/kg/día cada 24 h. Máximo 500 mg/dosis

- Amoxicilina-clavulánico durante 10 días - 40 mg/kg/día cada 8 h. Máximo $1 \mathrm{~g}$ cada 24 h

- Penicilina G benzatina, inyección única intramuscular profunda. Misma dosis que la indicada en la Tabla 4

- Más rifampicina 20 mg/kg/día cada 12 h. Máximo 600 mg/ día, los últimos 4 días

- Penicilina V (fenoximetilpenicilina) durante 10 días. Misma dosis que la indicada en al Tabla 4

- Más rifampicina 20 mg/kg/día cada 12 h. Máximo 600 mg/ día, los últimos 4 días

- Cefadroxilo durante 10 días. 30 mg/kg/día cada 12 h. Máximo $1 \mathrm{~g}$ cada $24 \mathrm{~h}$

- Más rifampicina 20 mg/kg/día cada 12 h. Máximo 600 mg/ día, los últimos 4 días

EbhGA = estreptococo beta hemolítico del grupo $\mathrm{A}$.

Tabla 10: Criterios diagnósticos del síndrome PFAPA.

1. Episodios recurrentes de fiebre de inicio antes de los 5 años

2. Síntomas constitucionales en ausencia de infección de vías respiratorias altas con al menos uno de los tres siguientes: estomatitis aftosa, linfadenitis cervical y/o faringitis

3. Exclusión de neutropenia cíclica

4. Completamente asintomático entre episodios

5. Crecimiento y desarrollo normales

PFAPA = acrónimo del inglés periodic fever, adenopathy, pharyngitis and aphthous stomatitis.

erradica la bacteria en $100 \%$ de los casos. Se trata de un estado de benignidad. ${ }^{73}$

La prevalencia del estado de portador puede durar meses y varía según áreas geográficas, afectando de $10-40 \%$ de los niños escolares sanos durante el invierno y primavera. ${ }^{1,2,5,74}$ Por ello, no se recomienda tratar los fracasos bacteriológicos ni el estado de portador de EbhGA, salvo en casos muy concretos $^{1,2,23,75}$ (Tabla 8) (BII).

Para los casos de portadores o fracasos bacteriológicos que precisen tratamiento, se han empleado algunos antibióticos ${ }^{1,53}$ que se muestran en la Tabla 9 , sin que exista suficiente evidencia científica para 
Rev Latin Infect Pediatr. 2021; 34 (2): 62-72

recomendar un tratamiento de elección concreto. La penicilina es ineficaz tanto por vía oral como intramuscular. La azitromicina en dosis altas se ha mostrado como la opción más efectiva en algún estudio por su excelente concentración intracelular, siempre que no haya sido administrada previamente ${ }^{63,76}$ (BII). En cuanto a la amigdalectomía, se recomienda cuando existen más de siete episodios de FAA por EbhGA documentadas y bien tratadas en un año, más de cinco en cada uno de los dos años anteriores o más de tres en cada uno de los tres años anteriores. En cada episodio debe presentarse al menos uno de los siguientes cuatro criterios: fiebre ( $>38.3$ ${ }^{\circ} \mathrm{C}$ ), adenopatía cervical, exudados amigdalares, o evidencia de infección por EbhGA. En las FAA de repetición con cultivos persistentemente negativos debe plantearse el diagnóstico del síndrome PFAPA (acrónimo del inglés periodic fever, adenopathy, pharyngitis and aphthous stomatitis), cuyos criterios diagnósticos ${ }^{77-79}$ se muestran en la Tabla 10. En la actualidad no existe ninguna vacuna para prevenir la FAA por EbhGA.

\section{REFERENCIAS}

1. Álvez González F, Sánchez Lastres JM. Faringoamigdalitis aguda. Protocolos de Infectología de la Asociación Española de Pediatría. [Consultado 1/7/11]. Disponible en: http://www. aeped.es/documentos/protocolos-infectologia

2. Wessels MR. Clinical practice. Streptococcal pharyngitis. N Engl J Med. 2011; 364: 648-655.

3. Álvez González F. Faringitis estreptocócica en niños menores de 2 años. An Pediatr (Barc). 2008; 68: 525-539.

4. Matas L, Méndez M, Rodrigo C, Ausina V. Diagnóstico de las faringitis estreptocócicas. Enferm Infecc Microbiol Clin. 2008; 26: 14-18.

5. Shaikh N, Leonard E, Martin JM. Prevalence of streptococcal pharyngitis and streptococcal carriage in children: a metaanalysis. Pediatrics. 2010; 126: e557-e564.

6. Flores Mateo G, Conejero J, Grenzner Martinel E, Baba Z, Dicono S, Echasabal M et al. Early diagnosis of streptococcal pharyngitis in paediatric practice: validity of a rapid antigen detection test. Aten Primaria. 2010; 42: 356361.

7. Gerber MA, Baltimore RS, Eaton CB, Gewitz M, Rowley $\mathrm{AH}$, Shulman ST et al. Prevention of rheumatic fever and diagnosis and treatment of acute Streptococcal pharyngitis: a scientific statement from the American Heart Association Rheumatic Fever. Endocarditis, and Kawasaki Disease Committee of the Council on Cardiovascular Disease in the Young, the Interdisciplinary Council on Functional Genomics and Translational Biology, and the Interdisciplinary Council on Quality of Care and Outcomes Research: endorsed by the American Academy of Pediatrics. Circulation. 2009; 119: 1541-1551.

8. Domínguez O, Rojo $\mathrm{P}$, De las Heras S, Folgueira D, Ruíz Contreras J. Clinical presentation characteristics of pharyngeal adenovirus infections. Pediatr Infect Dis J. 2005; 24: 733-734.

9. Carbonero Celis MJ, Torronteras Santiago R, Cintado Bueno C. Mononucleosis infecciosa: estudio en niños hospitalizados. An Esp Pediatr. 1999; 51: 664-666.

10. Hervás Ángulo A, Arizcuren Domeño MA, Tiberio López G, Oteiza Olaso J. Características clínico-analíticas y complicaciones de pacientes con mononucleosis infecciosa derivados desde atención primaria a atención especializada. Aten Primaria. 2003; 32: 311.

11. Santiago Sánchez Mateos JL, Aboín González S, García Millán C, Jaén Olasolo P. Mononucleosis infecciosa. Med Clin (Barc). 2008; 130: 520.

12. Bisno AL. Acute pharyngitis. N Engl J Med. 2001; 344: 205211.

13. Cooper RJ, Hoffman JR, Barlett JG, Besser RE, Gonzales $\mathrm{R}$, Hickner JM et al. Principles of appropriate antibiotic use for acute pharyngitis in adults: background. Ann Intern Med. 2001; 134: 509-517.

14. Snow V, Mottur-Pilson C, Cooper RJ, Hoffman JR. Principles of appropriate antibiotic use for acute pharyngitis in adults. Ann Intern Med. 2001; 134: 506-508.

15. Centor RM, Witherspoon JM, Dalton HP, Brody CE, Link $\mathrm{K}$. The diagnosis of strep throat in adults in the emergency room. Med Decis Making. 1981; 1: 239-246.

16. Mclsaac WJ, Kellner JD, Aufricht P, Vanjaka A, Low DE. Empirical validation of guidelines for the management of pharyngitis in children adults. JAMA. 2004; 291: 1587-1595 [Erratum, JAMA 2005; 294: 2700].

17. Ruíz-Aragón J, Rodríguez López R, Molina Linde JM. Evaluación de los métodos rápidos para la detección de Streptococcus pyogenes. Revisión sistemática y metaanálisis. An Pediatr (Barc). 2010; 72: 391-402.

18. Mayes T, Pichichero ME. Are follow-up throat cultures necessary when rapid antigen detection tests are negative for group A streptococci? Clin Pediatr (Phila). 2001; 40: 191195.

19. Cohen R, Levy C, Ovetchkine P, Boucherat M, Weil-Olivier C, Gaudelus J et al. Evaluation of streptococcal clinical scores, rapid antigen detection tests and cultures for childhood pharyngitis. Eur J Pediatr. 2004; 163: 281-282.

20. Gerber MA, Shulman ST. Rapid diagnosis of pharyngitis caused by group A streptococci. Clin Microbiol Rev. 2004; 17: $571-580$.

21. Jaggi P, Shulman ST, Group A. Streptococcal infections. Pediatr Rev. 2006; 27: 99-105.

22. Sarikaya S, Aktas C, Ay D, Cetin A, Celikmen F, Sensitivity. Specificity of rapid antigen detection testing for diagnosing pharyngitis in the emergency department. Ear Nose Throat J. 2010; 89: 180-182.

23. Fernández-Cuesta Valcarce MA. Faringitis aguda. En: Hernández Merino A, Bravo Acuna J, Merino Moíña M, Ramos Amador JT, Rojo Conejo P, Ruiz Contreras J, editores. Guía ABE. Infecciones en pediatría. Madrid: Exlibris Ediciones SL, Asociación Española de Pediatría de Atención Primaria; 2010. pp. 153-158.

24. Herranz B, Rodríguez-Salinas E, Orden B. Utilidad de las técnicas de diagnóstico rápido para la detección de Streptococcus pyogenes. An Pediatr Cont. 2007; 5: 92-95.

25. Denny FW, Wannamaker LW, Brink WR, Rammelkamp Jr $\mathrm{CH}$, Custer EA. Prevention of rheumatic fever; treatment of the preceding streptococcic infection. J Am Med Assoc. 1950; 143: 151-153. 
Rev Latin Infect Pediatr. 2021; 34 (2): 62-72

26. Catanzaro FJ, Stetson CA, Morris AJ, Chamovitz R, Rammelkamp Jr CH, Stolzer BL et al. The role of the streptococcus in the pathogenesis of rheumatic fever. Am J Med. 1954; 17: 749-756.

27. Potter EV, Svartman M, Mohammed I, Cox R, Poon-King T, Earle DP. Tropical acute rheumatic fever and associated streptococcal infections compared with concurrent acute glomerulonephritis. J Pediatr. 1978; 92: 325-333.

28. Randolph MF, Gerber MA, De Meo KK, Wright L. Effect of antibiotic therapy on the clinical course of streptococcal pharyngitis. J Pediatr. 1985; 106: 870-875.

29. Krober MS, Bass JW, Michaels GN. Streptococcal pharyngitis: Placebo-controlled double blind evaluation of clinical response to penicillin therapy. JAMA. 1985; 253: 1271-1274.

30. Pichichero ME, Disney FA, Talpey WB, Green JL, Francis AB, Roghmann $\mathrm{KJ}$ et al. Adverse beneficial effect of immediate treatment of group A -hemolytic streptococcal pharyngitis with penicillin. Pediatr Infect Dis J. 1987; 6: 635-643.

31. Snellman LW, Stang HJ, Stang JM, Johnson DR, Kaplan EL. Duration of positive throat cultures for group A streptococci after initiation of antibiotic therapy. Pediatrics. 1993; 91: 1166-1170.

32. Bisno AL, Gerber MA, Gwaltney JM, Kaplan EL, Schwartz $\mathrm{RH}$. Practice guidelines for the diagnosis and management of group A streptococcal pharyngitis. Clin Infect Dis. 2002; 35: 113-125.

33. Robertson KA, Volmink JA, Mayosi EM. Antibiotics for the primary prevention of acute rheumatic fever: a metaanalysis. BMC Cardiovasc Disord. 2005; 5: 11.

34. Pichichero M, Casey J. Comparison of European and U.S. results for cephalosporin versus penicillin treatment of group A streptococcal tonsillopharyngitis. Eur J Clin Microbiol Infect Dis. 2006; 25: 354-364.

35. Del Mar CB, Glasziou PP, Spinks AB. Antibiotics for sore throat. Cochrane Database Syst Rev. 2006; 4: CD000023.

36. Alcaide ML, Bisno AL. Pharyngitis epiglottitis. Infect Dis Clin N Am. 2007; 21: 449-469.

37. Shulman ST, Tanz RR, Dale JB, Beall B, Kabat W, Kabat K et al. Seven-year surveillance of North American pediatric group A streptococcal pharyngitis isolates. Clin Infect Dis. 2009; 49: 78-84.

38. Carapetis J, Steer A. Prevention of rheumatic fever. Pediatr Infect Dis J. 2010; 29: 91-92.

39. Álvez F. Uso racional de antibióticos en las infecciones más comunes de los niños. An Pediatr Contin. 2010; 8: 221-230.

40. Peñalba Citores AC, Riaño Méndez B, Marañón Pardillo R, Míguez Navarro C, Vázquez López P, Guerrero Soler MM et al. Incidencia de faringitis estreptocócica. An Pediatr (Barc). 2007; 67: 220-224.

41. Álvez F, Martín C. Infecciones por estreptococo beta hemolítico grupo A (EBGHA) y sus complicaciones. Estado de portador y recurrencias. En: Gómez Campedrá JA, Jiménez Ferreros L, Álvarez Calatayud G, editores. Patología aguda ORL en pediatría. Madrid: GlaxoSmithKline; 2004. pp. 83-96.

42. Coonan KM, Kaplan EL. In vitro susceptibility of recent North American group A streptococcal isolates to eleven oral antibiotics. Pediatr Infect Dis J. 1994; 13: 630-635.

43. Horn DL, Zabriskie JB, Austrian R, Cleary PP, Ferretti JJ, Fischetti VA et al. Why have group A streptococci remained susceptible to penicillin: Report on a simposium. Clin Infect Dis. 1998; 26: 1341-1345.
44. Van Driel ML, De Sutter Al, Keber N, Habraken H, Christiaens T. Different antibiotic treatments for group A streptococcal pharyngitis. Cochrane Database Syst Rev. 2010; 6: CD004406.

45. Baltimore RS. Re-evaluation of antibiotic treatment of streptococcal pharyngitis. Curr Opin Pediatr. 2010; 22: 7782.

46. Curtin-Wirt C, Casey JR, Murray PC, Cleary CT, Hoeger WJ, Marsocci SM et al. Efficacy of penicillin vs amoxicillin in children with Group A beta hemolytic streptococcal tonsillopharyngitis. Clin Pediatr (Phila). 2003; 42: 219-225.

47. Clegg HW, Ryan AG, Dallas SD, Kaplan EL, Johnson DR, Norton $\mathrm{HJ}$ et al. Treatment of streptococcal pharyngitis with once-daily compared with twice-daily amoxicillin: a noninferiority trial. Pediatr Infect Dis J. 2006; 25: 761-767.

48. Lennon DR, Farrel E, Martin DR, Stewart JM. Once-daily amoxicillin versus twice daily penicillin $\mathrm{V}$ in group $\mathrm{A}$ betahemolytic streptococcal pharyngitis. Arch Dis Child. 2008; 93: 474-478.

49. Ochoa Sangrador C, Vilela Fernández M, Cueto Baelo M, Eiros Bouza JM, Inglada Galiana L. Adecuación del tratamiento de la faringoamigdalitis aguda a la evidencia científica. An Pediatr (Barc). 2003; 59: 31-40.

50. Casaní Martínez C, Calvo Rigual F, Peris Vidal A, Álvares de Lavida Mulero T, Díez Domingo J, Graullera Millas M et al. Encuesta sobre el uso racional de antibióticos en atención primaria. An Pediatr (Barc). 2003; 58: 10-16.

51. Durán Fernández Feijoo $C$, Marqués Ercilla $S$, Hernández Bou S, Trenchs Sainz de la Maza V, García García JJ, Luaces Cubells C. Calidad de la prescripción antibiótica en un servicio de urgencias pediátrico hospitalario. An Pediatr (Barc). 2010; 73: 115-120.

52. Albañil Ballesteros MR, Calvo Rey C, Sanz Cuesta T. Variación de la prescripción de antibióticos en atención primaria. An Esp Pediatr. 2002; 57: 420-426.

53. Kaplan EL, Johnson DR. Eradication of group A streptococci from the upper respiratory tract by amoxicillin with clavulanate after oral penicillin $\mathrm{V}$ treatment failure. J Pediatr. 1988; 113: 400-403.

54. Casey JR, Pichichero ME. Meta-analysis of cephalosporin versus penicillin treatment of group A streptococcal tonsillopharyngitis in children. Pediatrics. 2004; 113: 866882.

55. Kaplan EL, Oakes JM, Johnson DR. Unexpected individual clinical site variation in eradication rates of group a Streptococci by penicillin in multisite clinical trials. Pediatr Infect Dis J. 2007; 26: 1110-1116.

56. Shulman ST, Gerber MA. So what's wrong with penicillin for strep throat? Pediatrics. 2004; 113: 1816-1819.

57. Álvez F. Eficacia de la penicilina en el tratamiento de las faringoamigdalitis estreptocócicas. An Pediatr Contin. 2004; 2: $105-117$.

58. Pichichero ME. A review of evidence supporting the American Academy of Pediatrics recommendation for prescribing cephalosporin antibiotics for penicillin-allergic patients. Pediatrics. 2005; 115: 1048-1057.

59. Perez-Trallero E, Urbieta M, Montes M, Ayestaran I, Marimon JM. Emergence of Streptococcus pyogenes strains resistant to erythromycin in Gipuzkoa, Spain. Eur J Clin Microbiol Infect Dis. 1998; 17: 25-31.

60. Orden B, Perez-Trallero E, Montes M, Martínez R. Erythromycin resistance of Streptococcus pyogenes in Madrid. Pediatr Infect Dis J. 1998; 17: 470-473. 
Rev Latin Infect Pediatr. 2021; 34 (2): 62-72

61. Betriu C, Casado MC, Gómez M, Sanchez A, Palau $\mathrm{ML}$, Picazo JJ. Incidence of erythromycin resistance in Streptococcus pyogenes: a 10-year study. Diagn Microbiol Infect Dis. 1999; 33: 255-260.

62. Pérez-Trallero E, Martín-Herrero JE, Mazón A, GarcíaDelafuente C, Robles P, Iriarte $\mathrm{V}$ et al. Antimicrobial resistance among respiratory pathogens in Spain: latest data and changes over 11 years (1996-1997 to 2006-2007). Antimicrob Agents Chemother. 2010; 54: 2953-2959.

63. Casey JR, Pichichero ME. Higher dosages of azithromycin are more effective in treatment of group A streptococcal tonsillopharyngitis. Clin Infect Dis. 2005; 40: 1748-1755.

64. Cohen R, Reinert P, De La Rocque F, Levy C, Boucherat M, Robert $\mathrm{M}$ et al. Comparison of two dosages of azithromycin for three days versus penicillin $V$ for ten days in acute group A streptococcal tonsillopharyngitis. Pediatr Infect Dis J. 2002; 21: 297-303.

65. Cohen R. Defining the optimum treatment regimen for azithromycin in acute tonsillopharyngitis. Pediatr Infect Dis J. 2004; 23: 129-134.

66. Richter SS, Heilmann KP, Beekmann SE, Miller NJ, Miller AI, Rice CL et al. Macrolide-resistant Streptococcus pyogenes in the United States, 2002-2003. Clin Infect Dis. 2005; 41: 599-608.

67. Tanz RR, Shulman ST, Shortridge VD, Kabat W, Kabat K, Cederlund $\mathrm{E}$ et al. Community-based surveillance in the United States of macrolide-resistant pediatric pharyngeal group A streptococci during 3 respiratory disease seasons. Clin Infect Dis. 2004; 39: 1794-1801.

68. Kim S, Yong Lee N. Antibiotics resistance and genotypic characteristics of group A streptococci associated with acute pharyngitis in Korea. Microb Drug Resist. 2004; 10: 300305.

69. Tamayo J, Pérez-Trallero E, Gómez-Garcés JL, Alós JI. Resistance to macrolides, clindamycin and telithromycin in Streptococcus pyogenes isolated in Spain during 2004. J Antimicrob Chemoter. 2005; 56: 780-782.

70. Rodríguez D, Danés I. Nuevas pautas de antibioterapia en la faringoamigdalitis aguda. Med Clin (Barc). 2001; 117: 115-116.

71. Falagas ME, Vouloumanou EK, Matthaiou DK, Kapaskelis AM, Karageorgopoulos DE. Effectiveness and safety of short-course vs long-course antibiotic therapy for group a beta hemolytic streptococcal tonsillopharyngitis: a metaanalysis of randomized trials. Mayo Clin Proc. 2008; 83: 880-889.

72. Markowitz M, Gerber MA, Kaplan EL. Treatment of streptococcal pharyngotonsillitis: reports of penicillin's demise are premature. J Pediatr. 1993; 123: 679-685.

73. Kaplan EL. The group A streptococcal upper respiratory tract carrier state: an enigma. J Pediatr. 1980; 97: 337-345.

74. Schwartz RH, Wientzen RL Jr, Pedreira F, Feroli EJ, Mella GW, Guandolo VL. Penicillin V for group A streptococcal pharyngotonsillitis. A randomized trial of seven vs ten days' therapy. JAMA. 1981; 246: 1790-1795.

75. Gerber MA. Treatment failures and carriers: perception or problems? Pediatr Infect Dis J. 1994; 13: 576-579.

76. Kaplan EL, Chhatwal GS, Rohde M. Reduced ability of penicillin to eradicate ingested group A streptococci from epithelial cells: clinical and pathogenic implications. Clin Infect Dis. 2006; 43: 1398-1406.

77. Marshall GS, Edwards KM, Butler J, Lawton AR. Syndrome of periodic fever, pharyngitis, and aphthous stomatitis. $J$ Pediatr. 1987; 110: 43-46.

78. Feder HM, Salazar JC. A clinical review of 105 patients with PFAPA (a periodic fever syndrome). Acta Paediatr. 2010; 99: 178-184.

79. Calvo Rey C, Soler-Palacín P, Muñoz RM, Lozano JS, López JA, Aróstegui JI et al. Documento de Consenso de la Sociedad de Infectología Pediátrica y la Sociedad de Reumatología Pediátrica sobre el diagnóstico diferencial y el abordaje terapéutico de la fiebre recurrente. An Pediatr (Barc). 2011; 74: e1-16.

Financiamiento: Ninguno.

Conflicto de intereses: Ninguno.

Correspondencia:

Roi Piñeiro Pérez

E-mail: roipineiro@telefonica.net 